\title{
A Rare Case Report of Hypoplasia of Liver Lobe with Ectopia of Gall Bladder- Is Small Liver Lobe Always Pathological?
}

\section{ABSTRACT}

Hypoplasia of right lobe of the liver with secondary ectopia of gall bladder is one of the rare morphological developmental anomalies of liver. Frequently, it is also associated with other hepatobiliary anomalies. Patients with such anomalies are more prone to surgical complications due to altered anatomical positions and are also at risk to develop cholecystitis, gall stones, and portal hypertension. The knowledge of such liver anomalies is important for radiologists to avoid surgical and medical pitfalls. Here, the authors report a rare case of hypoplasia of right lobe of liver in a 74-year-old female patient who had no clinical features of liver or biliary disease and with normal liver function test. It was incidentally detected in her Computed Tomography (CT) pulmonary angiogram and further confirmed in Ultrasound (US) and Magnetic Resonance Imaging (MRI).

\section{CASE REPORT}

A 74-year-old female patient presented with breathlessness for one week with associated tachycardia for which CT angiogram was done to rule out pulmonary thromboembolism. The liver function test was within normal limits. Clinically, there were no features of chronic liver disease.

The upper abdominal sections of the CT pulmonary angiogram showed small right lobe and enlarged left lobe with macrolobulations in the liver surface [Table/Fig-1]. US abdomen and Doppler were performed to evaluate the liver. US/Doppler study of the liver showed small right lobe and enlarged left lobe, with normal liver echotexture [Table/Fig-2]. The main and left portal vein was normal in caliber, colour flow, and spectral pattern. The right portal vein was small in caliber with normal colour flow and spectral pattern. There were no portosystemic collaterals.

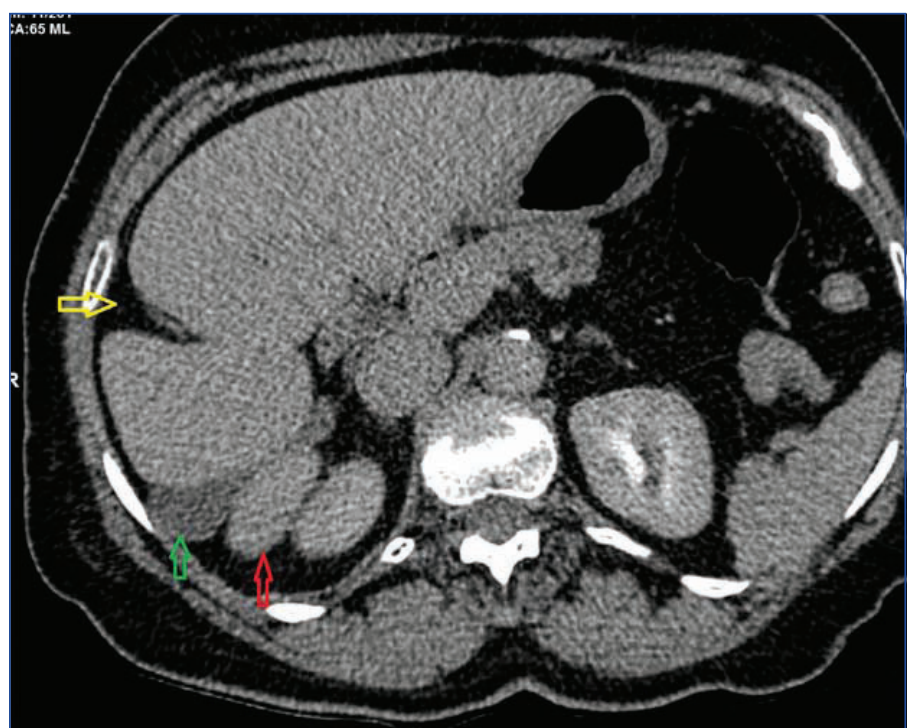

[Table/Fig-1]: Computed Tomography axial sections of the liver shows markedly small sized right lobe (red arrow) with enlarged left lobe and posterolaterally placed gall bladder (green arrow). The fissure for ligamentum Teres is marked by the yellow arrow.

The patient underwent $\mathrm{MRl}$ abdomen with dynamic contrast imaging which showed a markedly small right lobe with enlarged left lobe of the liver. The small right lobe was isointense to the left

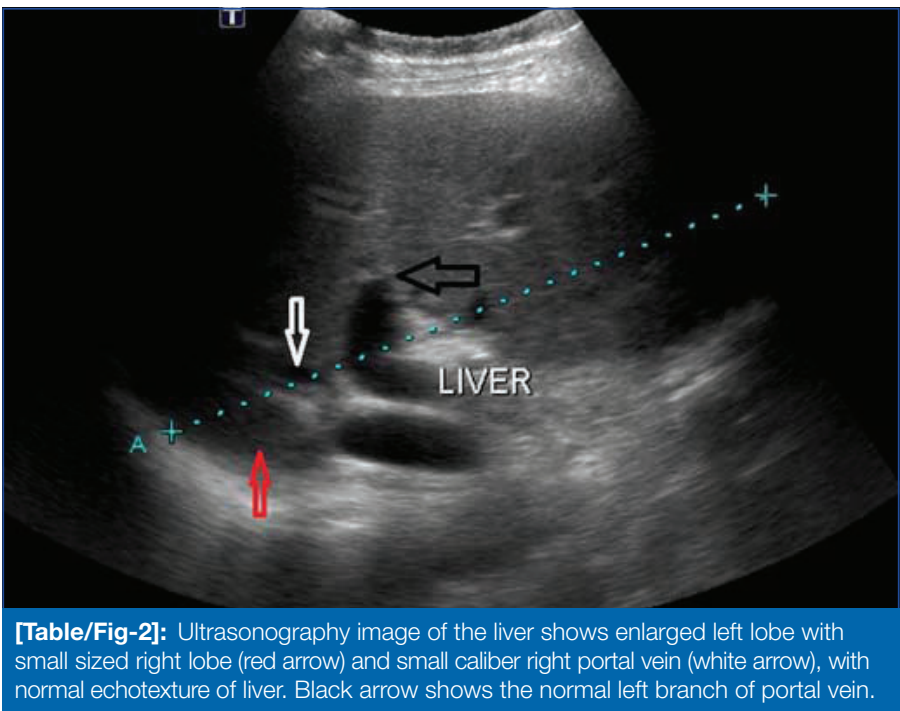

lobe on all sequences [Table/Fig-3a]. The main portal vein (14 mm) and its left branch $(12 \mathrm{~mm}$ ) were normal in caliber. The right portal vein was diffusely small in caliber $(3 \mathrm{~mm})$ as shown in [Table/Fig3b]. There were no portosystemic collaterals. Two hepatic veins (superior and inferior) were seen draining the small right lobe, which in turn were draining into the middle hepatic vein and IVC. The middle and left hepatic veins were normal. No evidence of any focal lesions in liver parenchyma. Intrahepatic biliary radicles were not dilated.
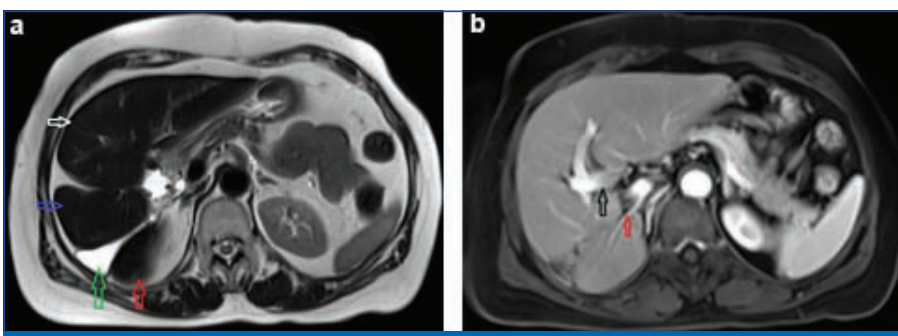

[Table/Fig-3]: a) T2 weighted axial image of the liver shows posterolaterally placed gall bladder (green arrow), small sized right lobe (red arrow), and enlarged medial (white arrow) and lateral (blue arrow) segments of left lobe of the liver. b) Post-contrast T1 weighted fat suppressed images of the liver shows small sized right lobe of liver with small caliber right portal vein (red arrow), enlarged left lobe with normal left branch of portal vein (black arrow). 
Gall bladder was seen deviated to the right postero-lateral aspect just posterior to the enlarged left lobe and anterior to the small right lobe [Table/Fig-3b]. No cholelithiasis or features of cholecystitis were noted.

Hence, with a small sized right lobe of liver with a very small caliber right portal vein and right hepatic vein and ectopia of gall bladder and after excluding other acquired causes for small sized right lobe, a diagnosis of hypoplasia of right lobe of the liver was made.

\section{DISCUSSION}

Lobar aplasia or hypoplasia of liver is a very rare congenital anomaly with incidence of $0.005 \%$ in autopsy studies [1]. The morphological developmental anomalies of the liver include agenesis, aplasia, or hypoplasia [2]. Agenesis is the absence of a lobe with replacement by fibrous tissue, aplasia is small lobe with abnormal structure and hypoplasia is small lobe with normal structure. Hypoplasia of right lobe is sometimes associated with ectopy of gall bladder [3] or other bile duct anomalies.

Kakitsubata $Y$ et al., have reported two case reports of hypoplasia of right hepatic lobe associated with ectopy of gall bladder [4]. Similar to patient in present report, there were no other associated biliary complications. Kabaroudis A et al., have reported a case of hypoplasia of right hepatic lobe combined with a floating gall bladder which was incidentally detected in CT scan done for staging colonic cancer [1]. There were also features of chronic cholecystitis with diffusely calcified gall bladder walls. No features of acute or chronic cholecystitis or cholelithiasis were seen in the current patient. Many other associated anomalies like persistent right umbilical vein [5], diaphragmatic eventration [6], intrathoracic kidney, right diaphragmatic hernia, cysts in biliary system and veno-venous shunts in liver [7] are reported in the literature. This patient had no associated anomalies.

The majority of morphological anomalies of liver remain clinically silent, with most of them being discovered as incidental findings on US, CT, MRI, or even intraoperatively [6]. Patients with these anomalies are more prone for cholecystitis, cholelithiasis, biliary strictures, gall bladder carcinoma, and portal hypertension $[8,9]$. Gall bladder can be retrohepatic, suprahepatic, or transverse in location in right lobe hypoplasia. In this patient, gall bladder was positioned transversely (right posterior) in the right dorso-lateral aspect located between the small right lobe and enlarged left lobe with no cholelithiasis or cholecystitis. Alicioglu $B$ reported a case of right hepatic lobe hypoplasia associated with the abnormal course of the portal vein and common bile duct which are more prone for injuries during surgical and interventional procedures [10]. Similarly in this patient, the main portal vein and common bile duct were seen to be coursing abnormally, being placed more posterolaterally. Hence, it is important to be aware of the other associated anomalies and anomalous positions of the perihepatic structures in cases of hypoplasia of liver lobes.

A systematic comparative analysis of radiological features of developmental anomalies of the right hepatic lobe was done by Lian LL et al., and they concluded that such liver abnormalities have no clinical hallmark and there was no statistical difference in the efficiency of CT, MRI, and US in identifying the characteristics of these developmental anomalies of the right lobe of liver [11].

\section{CONCLUSION(S)}

Knowledge of the morphological liver anomalies is important to help in hepatobiliary surgical planning, to avoid inadvertent iatrogenic injuries and, to differentiate them from acquired causes of small liver lobe to avoid medical pitfalls. The patients with such anomalies should also be subjected to periodical screening for secondary complications.

\section{REFERENCES}

[1] Kabaroudis A, Papaziogas B, Atmatzidis K, Argiriadou E, Paraskevas A, Galanis I, et al. Hypoplasia of the right hepatic lobe combined with a floating gallbladder. Acta Chir Belg. 2003;103(4):425-27.

[2] Hassan GM, Sliem HA, Ellethy AT. Hepatic embryonic development and anomalies of the liver. J Gastroenterol Hepatol Res. 2013;2(4):489-93.

[3] Sethi SK, Solanki RS. A case report- hypoplastic right hepatic lobe- A rare anomaly. Indian J Radiol Imaging. 2004;14(1):53-54. https://www.msjonline.org/ index.php/ijrms/article/view/1270.

[4] Kakitsubata Y, Kakitsubata S, Watanabe K. Hypoplasia of the right hepatic lobe with ectopy of the gallbladder. Clin Imaging. 1995;19(2):85-87.

[5] Shankar N, Rabi S. A rare case of combined hypoplasia of the right lobe of the liver and a persistent right umbilical vein. Eur J Anat. 2020;9(1):55-57.

[6] Swarup MS, Bhatt S, Tandon A, Mandal S. Segmental hypoplasia of liver: The importance of radiologic recognition and reporting despite masterly inactivity. Egypt J Radiol Nucl Med. 2018;49(1):01-03.

[7] Hashimoto M, Sato K, Endo K. Right hepatic agenesis associated with unusual anatomic findings on hepatic venography. Radiat Med. 1997;15(4):223-25.

[8] Inoue T, Ito Y, Matsuzaki Y, Okauchi Y, Kondo H, Horiuchi N, et al. Hypogenesis of right hepatic lobe accompanied by portal hypertension: Case report and review of 31 Japanese cases. J Gastroenterol. 1997;32(6):836-42.

[9] Hsu KL, Cheng YF, Ko SF. Hypoplastic right hepatic lobe with retrohepatic gallbladder complicated by hepatolithiasis and liver abscess: A case report. Hepatogastroenterology. 1997;44(15):803-07.

[10] Alicioglu B. Right liver lobe hypoplasia and related abnormalities. Pol J Radiol. 2015;80:503-05. doi: 10.12659/PJR.894658. eCollection 2015.

[11] Liang LL, Li HJ, Hu Y, Li A, Hu D, Li Z. Developmental anomalies of the right hepatic lobe: Systematic comparative analysis of radiological features. Open Life Sci. 2017;12(1):489-500.

PARTICULARS OF CONTRIBUTORS:

1. Senior Resident, Department of Radiology, Christian Medical College, Vellore, Tamil Nadu, India.

2. Professor, Department of Radiology, Christian Medical College, Vellore, Tamil Nadu, India.

NAME, ADDRESS, E-MAIL ID OF THE CORRESPONDING AUTHOR:

Dr. Anu Eapen,

Professor, Department of Clinical Radiology, Christian Medical College,

IDA Scudder Road, Vellore-632004, Tamil Nadu, India.

E-mail: anuepn@yahoo.com

\section{AUTHOR DECLARATION}

- Financial or Other Competing Interests: None

- Was informed consent obtained from the subjects involved in the study? Yes

- For any images presented appropriate consent has been obtained from the subjects. Yes
PLAGIARISM CHECKING METHODS: [Jain Het al.]

- Plagiarism X-checker: Aug 27, 2020

- Manual Googling: Oct 13, 2020

- iThenticate Software: Dec 21, 2020 (6\%)
ETYMOLOGY: Author Origin

Date of Submission: Aug 25, 2020 Date of Peer Review: Oct 15, 2020

Date of Acceptance: Nov 24, 2020 Date of Publishing: Apr 01, 2021 